\title{
Phylogenetic Positions of Clostridium chauvoei and Clostridium septicum Based on 16S rRNA Gene Sequences
}

\author{
PETER KUHNERT,* SELJA E. CAPAUL, JACQUES NICOLET, AND JOACHIM FREY \\ Institute of Veterinary Bacteriology, University of Bern, CH-3012 Bern, Switzerland
}

\begin{abstract}
The sequences of the 16S rRNA genes (rrs genes) of Clostridium chauvoei, the causative agent of blackleg in cattle, and the phenotypically related organism Clostridium septicum were determined. After amplification of 1,507-bp PCR fragments from the corresponding $r$ rs genes, the sequences were determined in a single round of sequencing by using conserved region primers. A sequence similarity analysis of the sequences revealed the close phylogenetic relationship of $C$. chauvoei and $C$. septicum in Clostridium cluster I (M. D. Collins, P. A. Lawson, A. Willems, J. J. Cordoba, J. Fernandez-Garayzabal, P. Garcia, J. Cai, H. Hippe, and J. A. E. Farrow, Int. J. Syst. Bacteriol. 44:812-826, 1994), which includes Clostridium carnis, Clostridium perfringens, Clostridium botulinum, and Clostridium tetani. We found that $99.3 \%$ of the nucleotides in the genes of $C$. chauvoei and $C$. septicum are identical.
\end{abstract}

Clostridium chauvoei, the etiologic agent of blackleg, is a very virulent pathogen of cattle that causes fever, depression, lameness, and a high level of mortality. Because of the similar courses of the diseases, blackleg was formerly frequently confused with malignant edema caused by Clostridium novyi and with anthrax caused by Bacillus anthracis. Symptoms similar to blackleg symptoms can be caused by Clostridium septicum, $C$. novyi, Clostridium perfringens, and Clostridium sordellii (1). C. chauvoei is a gram-positive, anaerobic, spore-forming bacterium that has strong hemolytic activity. The genus Clostridium consists of organisms whose $\mathrm{G}+\mathrm{C}$ contents vary from 22 to 55 mol\% (5). Many new Clostridium species have been described recently; the genus now contains about 100 species, some of which are pathogenic and some of which are nonpathogenic (8). Many pathogenic clostridia, including $C$. chauvoei, have been shown to produce toxins which seem to be responsible for pathogenicity (for a review see reference 4). Despite the extensive work on the phylogeny of the species belonging to the genus Clostridium by Collins et al. (3), the phylogenetic positions of $C$. chauvoei and $C$. septicum have never been determined. Collins et al. (3) sequenced the $r r s$ genes encoding the $16 \mathrm{~S}$ rRNAs of 34 different clostridial species and determined their phylogenetic relationships to more than 80 previously determined clostridial $r r s$ sequences and $r r s$ sequences of representative species of other low-G+C-content gram-positive genera. Almost one-half of the clostridial species examined belonged to a phenotypically well-defined cluster designated cluster I. The remaining clostridial species exhibited considerable degrees of phylogenetic diversity and formed 19 clusters and four individual lines of descent.

In order to resolve the phylogenetic position of $C$. chauvoei, we determined its complete $r s$ gene sequence from DNA obtained by PCR amplification of genomic DNA from the $C$. chauvoei type strain and a field isolate. In parallel, we also determined the rrs gene sequence of the phenotypically very similar organism $C$. septicum.

Amplification of 16S ribosomal DNA. The strains used in this study were $C$. chauvoei ATCC $10092^{\mathrm{T}}$ ( $\mathrm{T}=$ type strain) and diagnostic field isolates of $C$. chauvoei, as well as $C$. sep-

\footnotetext{
${ }^{*}$ Corresponding author. Mailing address: Institute of Veterinary Bacteriology, Laenggass-Str. 122, CH-3012 Bern, Switzerland. Phone: 41-31-6312369. Fax: 41-31-6312634. Electronic mail address: Kuhnert (avbi.unibe.ch.
}

ticum ATCC $12464^{\mathrm{T}}$ and ATCC 8065 . All strains were grown in Schaedler broth (code no. CM 497; Oxoid, Basingstoke, United Kingdom) anaerobically at $37^{\circ} \mathrm{C}$ without shaking. Chromosomal DNA was prepared by the guanidinium thiocyanate method of Pitcher et al. (7). PCR were performed with a model PE9600 automated thermal cycler equipped with MicroAmp tubes (Perkin-Elmer Cetus, Norwalk, Conn.) by using a polymerase with proof-reading activity in order to avoid artificial sequence divergence. Directly sequencing the PCR product without further cloning increased the accuracy of the sequence determination. Each $50-\mu l$ reaction mixture contained $5 \mu \mathrm{l}$ of $10 \times$ PCR buffer, 20 pmol of each primer, $1 \mathrm{mM}$ deoxynucleoside triphosphate, $2.5 \mathrm{U}$ of Pwo DNA polymerase (Boehringer, Mannheim, Germany), and $100 \mathrm{ng}$ of genomic DNA. The PCR conditions were as follows: 35 cycles consisting of $94^{\circ} \mathrm{C}$ for $30 \mathrm{~s}, 54^{\circ} \mathrm{C}$ for $30 \mathrm{~s}$, and $72^{\circ} \mathrm{C}$ for $30 \mathrm{~s}$. A final extension step for $7 \mathrm{~min}$ at $72^{\circ} \mathrm{C}$ was also included. The primers used for PCR generation of $r s$ gene fragments were universal primer 16SUNI-L (Table 1) derived from the Escherichia coli $r r s$ sequence (2) and primer UNI16S-R (Table 1) derived from the rrs sequence of Clostridium botulinum (6), which was used to amplify the most $3^{\prime}$ part of the $C$. chauvoei $C$. septicum $r$ rs genes.

Sequencing. The PCR product was purified with a QIAquick PCR purification kit used according to the recommendations

TABLE 1. Primers used for amplification and/or sequencing of $r s$ DNA

\begin{tabular}{llc}
\hline \multicolumn{1}{c}{ Primer } & \multicolumn{1}{c}{ Sequence } & Location $^{a}$ \\
\hline 16SRNAI-S & 5' CTACGGGAGGCAGCAGTGGGG 3' & 341-361 \\
16SRNAII-S & 5' GTGTAGCGGTGAAATGCGTAG 3' & $682-702$ \\
16SRNSIII-S & 5' GCCTGGGGAGTACGGTCGCAAG 3' & $880-901$ \\
16SRNAIV-S & 5' GGTTAAGTCCCGCAACGAGCGC 3' & 1087-1108 \\
16SRNAV-S & 5' CCCCACTGCTGCCTCCCGTAG 3' & $361-341$ \\
16SRNAVI-S & 5' CTACGCATTCACCGCTACAC 3' & $702-682$ \\
16SRNSVII-S & 5' CTTGCGACCGTACTCCCCAGGC 3' & $901-880$ \\
16SRNAVIII-S & 5' GCGCTCGTTGCGGGACTTAACC 3' & $1108-1087$ \\
16SUNI-L & 5' AGAGTTTGATCATGGCTCAG 3' & $8-27$ \\
16SUNI-R & 5' GTGTGACGGGCGGTGTGTAC 3' & $1410-1391$ \\
UNI16S-R & 5' AAGGAGGTGATCCAGCCGCA 3' & $1540-1521^{\prime}$ \\
\hline
\end{tabular}

\footnotetext{
${ }^{a}$ The numbers indicate the positions relative to the $E$. coli $r r s$ sequence (accession number J01859).

${ }^{b}$ All of the primers except UNI16S-R, which was obtained from the $C$. botulinum sequence, are universal to clostridia and $E$. coli, as determined by $r r s$ comparisons.
} 


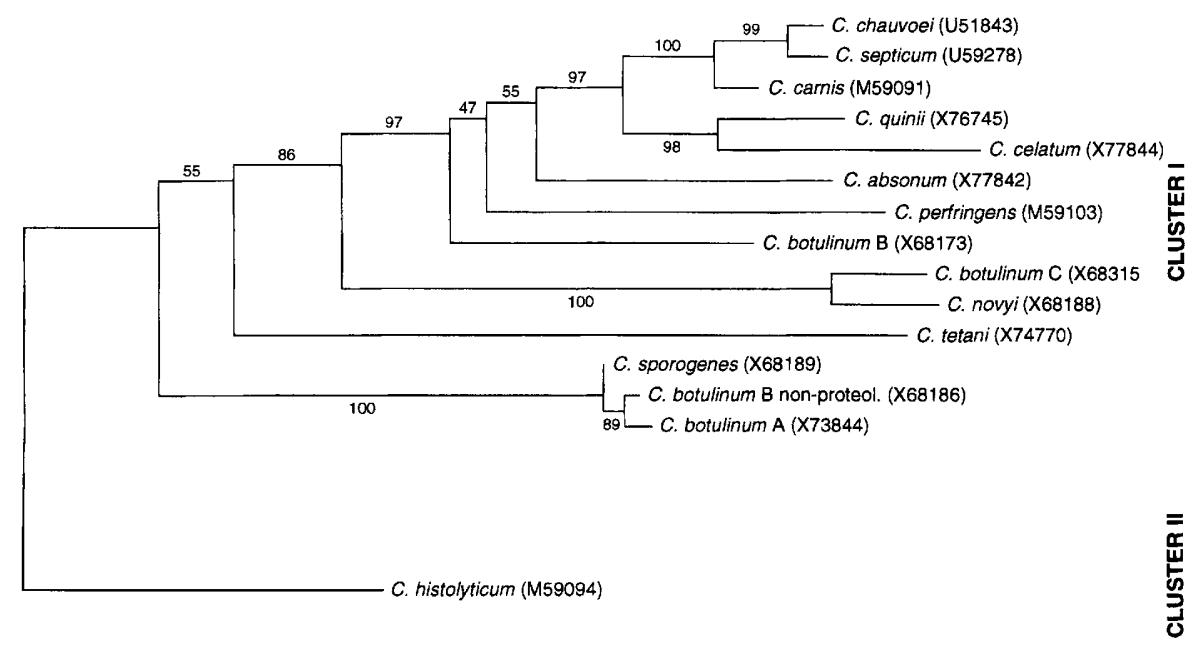

FIG. 1. Phylogenetic relationships of $C$. chauvoei and C. septicum to other clostridia based on 16S rRNA gene (rrs) sequences. The rrs sequences were trimmed, which resulted in fragments ranging from position 103 to position 1410 (E. coli numbering); thus, highly variable region V1 at the $5^{\prime}$ end was omitted. The GenBank accession numbers of the sequences of the $r s$ genes of the organisms are given in parentheses. Phylogenetic relationships were established with the Mega 1.02 program; corrected distances were calculated with the Jukes-Cantor algorithm, and a tree was constructed by using the neighbor-joining method. Bootstrap values were calculated from 1,000 trees. This combination of techniques resulted in the most similar tree structure compared with the tree used by Collins et al. (3), which allowed a direct comparison of results. $\mathrm{Bar}=1 \%$ sequence divergence.

of the manufacturer (QIAGEN, Hilden, Germany). The purified PCR product was then sequenced directly by using a Taq DyeDeoxy terminator cycle sequencing kit (ABI/PerkinElmer, Norwalk, Conn.) with approximately $300 \mathrm{ng}$ of template DNA per reaction mixture and 5 pmol of primer. The sequencing primers were designed by aligning the rrs sequences of $C$. botulinum types A, C, and E, C. novyi, Clostridium sporogenes, Clostridium histolyticum, C. perfringens, Clostridium cadaveris, and $C$. sordellii with the sequence of $E$. coli. By defining regions of sequence identity in all strains and choosing primers within these regions we were able to develop a sequencing strategy that resulted in complete sequencing of the rrs gene on both strands in one round. The primers which we used are probably universal for their sequences also match the $E$. coli rrs sequence. Since universal primers 16SUNI-L and 16SUNI-R only amplify a 1,400 -bp region of $r r s$, a primer that was used for C. botulinum (16) was chosen to produce a PCR fragment that extended an additional $100 \mathrm{bp}$ at the $3^{\prime}$ end. The PCR was performed with primers 16SUNI-L and UNI16S-R. The sequence was edited on both strands by using the Sequencher program (GeneCodes, Ann Arbor, Mich.).

In order to confirm the rrs sequence of the $C$. chauvoei type strain, we also sequenced the rrs gene of a field strain isolated from an animal which died of blackleg. The rrs sequences of strain ATCC $10092^{\mathrm{T}}$ and the field isolate were identical. Likewise, the rrs gene sequences of $C$. septicum ATCC $12464^{\mathrm{T}}$ and ATCC 8065 were identical.

Phylogenetic analysis. The rrs gene sequences of $C$. chauvoei and $C$. septicum exhibited a high level of similarity $(99.3 \%)$. In order to place these organisms phylogenetically, their $r r s$ sequences were compared with the rrs sequences of a variety of clostridial species. To do this as described previously (3), the region from position 103 to position 1410 (E. coli numbering) was used for the calculations; thus, extremely variable region $\mathrm{V} 1$ at the $5^{\prime}$ end of the rrs gene was omitted. From their habitats and biochemical properties we assumed that $C$. chauvoei and $C$. septicum probably belong to Clostridium cluster I. Therefore, we used representatives of this group to locate the phylogenetic positions of these organisms roughly. In this anal- ysis, in which proteolytic $C$. botulinum types A and B, C. botulinum type $C, C$. sporogenes, Clostridium tetani and $C$. perfringens were used, we found that on the basis of their rrs sequences $C$. chauvoei and $C$. septicum are phylogenetically located near $C$. perfringens. For fine phylogenetic positioning of $C$. chauvoei and $C$. septicum we included strains that are most closely related to $C$. perfringens in our final analysis, as well as the cluster II organism $C$. histolyticum as an outgroup. This comparison revealed that $C$. chauvoei and $C$. septicum are closely related to Clostridium carnis (Fig. 1). We found that the level of $r r s$ sequence similarity was $97 \%$ between $C$. chauvoei and $C$. carnis, as well as between $C$. septicum and $C$. carnis.

The close phylogenetic relationship between $C$. chauvoei and C. septicum is a molecular reflection of the phenotypic similarity of these organisms. $C$. chauvoei is relatively difficult to distinguish from $C$. septicum, which might cause problems in diagnosis, since $C$. septicum can cause symptoms very similar to blackleg symptoms. In this respect we hope to develop a specific identification system for $C$. chauvoei based on the rrs gene sequences presented in this paper. This could be done with PCR and/or restriction fragment length polymorphism, whereas there might be some limitations because of the few nucleotide differences. It might even be possible to sequence specific parts of the rrs gene in order to determine by sequence analysis the correct species isolated from clinical material. In this way our work should help improve diagnostic tools for $C$. chauvoei based on molecular genetics.

Nucleotide sequence accession numbers. The sequences which we determined have been deposited in the GenBank database under accession numbers U51843 (C. chauvoei) and U59278 (C. septicum).

We thank R. Zanoni for helpful discussions concerning phylogenetic analysis and M. Krawinkler for technical assistance.

This work was supported by the Foundation Research 3R (grant 37/92) and by the Priority Program Biotechnology of the Swiss National Science Foundation (grant 5002-038920). 


\section{REFERENCES}

1. Blaha, T. 1989. Applied veterinary epidemiology. Gustav Fischer Press, New York.

2. Carbon, P., C. Ehresmann, B. Ehresmann, and J. P. Ebel. 1979. The complete nucleotide sequence of the ribosomal 16-S RNA from Escherichia coli. Eur. J. Biochem. 100:399-410.

3. Collins, M. D., P. A. Lawson, A. Willems, J. J. Cordoba, J. FernandezGarayzabal, P. Garcia, J. Cai, H. Hippe, and J. A. E. Farrow. 1994. The phylogeny of the genus Clostridium: proposal of five new genera and eleven new species combinations. Int. J. Syst. Bacteriol. 44:812-826.

4. Hatheway, C. L. 1990 . Toxigenic clostridia. Clin. Microbiol. Rev. 3:66-98.

5. Hippe, H., J. R. Andreesen, and G. Gottschalk. 1991. The Genus Clostridium-nonmedical, p. 1800-1866. In A. Balows, H. G. Trüper, M. Dworkin, W.
Harder, and K.-H. Schleifer (ed.), The prokaryotes, 2nd ed. Springer-Verlag, New York.

6. Hutson, R. A., D. E. Thomson, and M. D. Collins. 1993. Genetic interrelationships of saccharolytic Clostridium botulinum types B, E and F and related clostridia as revealed by small-subunit rRNA gene sequences. FEMS Microbiol. Lett. 108:103-110.

7. Pitcher, D. G., N. A. Saunders, and R. J. Owen. 1989. Rapid extraction of bacterial genomic DNA with guanidium thiocyanate. Lett. Appl. Microbiol. 8:151-156.

8. Smith, L. D. S. 1991. The genus Clostridium-Medical, p. 1867-1878. In A. Balows, H. G. Trüper, M. Dworkin, W. Harder, and K.-H. Schleifer (ed.), The prokaryotes, 2nd ed. Springer-Verlag, New York. 